\title{
Cyclodextrin Based Nanoparticles For Improved Solubility and Drug Delivery
}

\author{
Devashish Pandey ${ }^{1} \cdot$ Vikas Singh Panwar $^{1} \cdot$ Himanshu Mishra ${ }^{1} \cdot$ Lokesh Adhikari $^{1} \bullet$ Mukesh $^{2}$ \\ Pandey ${ }^{1}$ Mona Semalty ${ }^{1 *}$ \\ ${ }^{1}$ Department of Pharmaceutical Science, H.N.B Garhwal University Campus, Tehri Garhwal-249161, Uttarakhand \\ *Corresponding Author Email: monasemalty@gmail.com
}

Received: 31.03.2021; Revised: 16.04.2021; Accepted: 19.04.2021

(C)Society for Himalayan Action Research and Development

\begin{abstract}
Biopharmaceutical properties (Solubility and permeability etc.) of the drugs are the majorfactorsresponsible for absorbing the drug.A huge fraction of pharmaceutical drugs belongs to classes II and IV, showing more lipophilic nature with lowsolubility. These drugs can be complexed with cyclodextrin for improving thebiopharmaceutical properties and drug delivery. Cyclodextrin is widely utilized inpharmaceutical formulations as an associate excipient to extend stability and solubility-cyclodextrin tends to form complexes, and self-aggregation property results to form nano or micro preparations. Nanopreparation of cyclodextrins can be used for drug delivery. This review deals with cyclodextrin inclusion complexes/ nanoparticles, their preparations, application, and drug delivery role.
\end{abstract}

Keywords: Cyclodextrin $\bullet$ Nanoparticles $\bullet$ Solubility $\bullet$ Drug Delivery $\bullet$ Complexes

\section{Introduction}

Solubility is defined as the most quantity of the solute that can be dissolved in a given solvent. The solubility of drugs decides further dissolution, the drug's disintegration, and finally to the drug's systematic absorption. Solubility can be enhanced by several methods as $\mathrm{pH}$ adjustment, cosolvency, particle size reduction, using surfactants such as cyclodextrin as preparation of inclusion complex of drug with cyclodextrin which founded less toxic and increased solubility of insoluble drugs such as reported by various studies by entrapping the drug in cyclodextrin Coltescu et al. (2020).

Cyclodextrin is a naturally occurring oligosaccharide that is used as a solubilizer in pharmaceutical formulations. Most drugs are lipophilic in nature, and their complex with hydrophilic CDs allow the formulation to stabilize in an aqueous solution. The amphiphilic nature of CD entraps more drug molecules as lipophilic and hydrophilic drugs get entrapped to it. CD can also be used to mask the odor, taste of the content and also prevent environmental factors Hadian et al. (2018), Hedges (2009), Loftsson et al. (2019).

Nanoscience is widely applied to every field of technology, even in pharmaceuticals; it has a broad role as its nanosize makes the drug more permeable and nanosize of formulation makes the drug easily cross the barriers. It can also be used for drug targeting, increasing drug efficiency, and reducing drug dumping and side effects. Cyclodextrin property of self-aggregation in aqueous solution utilized for nano molecules formulation Varan et al. (2017). Nanopreparation of CDs is prepared without any harmful chemical or reagent in it Peñalva et al. (2019).

Drug targeting increases therapeutic effect as well as reduces drug dumping and toxicity in the organ. Nanoparticle provides specific targeting which is safe to be used due to limited or low drug loading. $\mathrm{CD}$ increase drug loading of nanoparticle to carry a large amount of drug. HP- $\beta-C D$ increased saquinavir loading into polynanoparticles by providing a soluble drug reservoir Loftsson et al. (2005). 
This review presents an overview of $\mathrm{CD}$, nanoparticle-based $\mathrm{CD}$, different routes for administration.

\section{Cyclodextrin}

Cyclodextrins are cyclic oligosaccharides $(6 \mathrm{D}(+)$ glucopyranose units linked by $\alpha-(1,4)$ bonds) made up of starch by the action of enzymes produced by bacteria. The presence of glucopyranose units gives a bucket-like shape where the inner cavity is lipophilic, and the outer cavity is hydrophilic with hydroxyl groups Challa et al. (2005).

$\mathrm{CD}$ is used in many formulations to increase drugs' solubility to stabilize the compound, mask the content for its taste, odor, and entrap volatile oilsLoftsson et al. (2019). The complexing tendency of CD was studied and discovered in 1935 by Pringston. In 1950 its cyclic structure was explained by Frendenberg and Cramer,Wimmer (2003). CD was synthesized by substituting with a hydroxyl group to increase the water solubility of natural CD. CD can increase solubility for poorly soluble drugs and hamper the bioavailability of high soluble and permeable drugs Loftsson and Duchêne (2007). CD has an inner cavity as a lipophilic and an outer cavity as a hydrophilic outer surface, making CD amphiphilic and able to interact or form a complex with various guest molecules. CD complexes are studied to raise the solubility while also enhancing bioavailability and preventing atmospheric factors Mura (2014).

Guest molecule and $\mathrm{CD}$ are bounded with different forces like hydrogen bonding, van der wal forces, electrostatic interactions, dispersion force, and hydrophobic interactions improve complex ability. CD complexes are mainly studied to enhancenon-polar drugs' solubility, enhance bioavailability, and prevent any effect due to atmosphere factors Semalty et al. (2014a).

$\mathrm{CD}$ also coats the drug and can mask the odor and taste of the drug, which prevents evaporation and stabilizes volatile substances such as oil or any solvent. Liquid components can also be converted into free-flowing powder, which improves handling and eases to carry, pack, and store the formulation. According to a range of glucose monomers in cyclodextrin, they are categorized into three types (called as parent cyclodextrins):

1. $\alpha$-CD: Contain a six-membered sugar ring molecule.

2. $\beta$-CD: Contain a seven-membered sugar ring molecule.

3. $\gamma$-CD: Contain eight-membered sugar ring molecule. Akasha et al. (2014)

\section{Properties and structure of the cyclodextrin}

Cyclodextrin physicochemical properties are listed in Table 1 Szejtli (1998)

Table 1: Properties of parent cyclodextrins

\begin{tabular}{|c|c|c|c|}
\hline Property & $\alpha$-cyclodextrin & $\beta$-cyclodextrin & $\gamma$-cyclodextrin \\
\hline Formula & $\mathrm{C}_{36} \mathrm{H}_{60} \mathrm{O}_{30}$ & $\mathrm{C}_{42} \mathrm{H}_{70} \mathrm{O}_{35}$ & $\mathrm{C}_{48} \mathrm{H}_{80} \mathrm{O}_{40}$ \\
\hline $\begin{array}{l}\text { Present of } \\
\text { Glucopyranose units }\end{array}$ & 6 & 7 & 8 \\
\hline $\begin{array}{l}\text { Molecular weight } \\
\left(\mathrm{g} \mathrm{mol}^{-1}\right)\end{array}$ & 972 & 1135 & 1297 \\
\hline $\begin{array}{l}\text { Solubility in water at } \\
25^{\circ} \mathrm{C}(\% \mathrm{w} / \mathrm{v})\end{array}$ & 14.5 & 1.85 & 23.2 \\
\hline Outer diameter(§̊) & 14.6 & 15.4 & 17.5 \\
\hline $\begin{array}{l}\text { Diameter of cavity } \\
\text { (̊) }\end{array}$ & $4.7-5.3$ & $6-6.5$ & $7.5-8.3$ \\
\hline $\begin{array}{l}\text { Volume of cavity } \\
\operatorname{volume}\left(\AA^{\mathbf{3}}\right)\end{array}$ & 174 & 262 & 427 \\
\hline Height of torus $(\stackrel{\AA}{\mathbf{A}})$ & 7.9 & 7.9 & 7.9 \\
\hline pKat $25^{\circ} \mathrm{C}$ & 12.332 & 12.202 & 12.081 \\
\hline $\mathbf{M P}{ }^{\circ} \mathbf{C}$ & $>200^{\circ} \mathrm{C}$ & $>200^{\circ} \mathrm{C}$ & $>200^{\circ} \mathrm{C}$ \\
\hline Crystal water, wt \% & 10.2 & $13.2-14.5$ & $8.13-17.7$ \\
\hline
\end{tabular}


Cyclodextrins are insoluble in organic solvents such as ketones, chlorinated hydrocarbons, alcohol, ethers, aliphatic and aromatic hydrocarbons Wimmer (2003). CDs in acid are hydrolyzed very slowly, but they are stable and highly soluble in alkaline solution. CDs are watersoluble, and solubility increases with temperature rise. Comparatively, $\gamma$-CDs are more soluble than $\alpha \mathrm{CD}$ and $\beta-\mathrm{CD} ; \beta-\mathrm{CD}$ is least soluble. Solubility depends on the interaction of the hydroxyl group with water Hedges (2009). Cyclodextrin derivatives can be prepared by substituting hydrogen to primary and secondary hydroxyl groups.
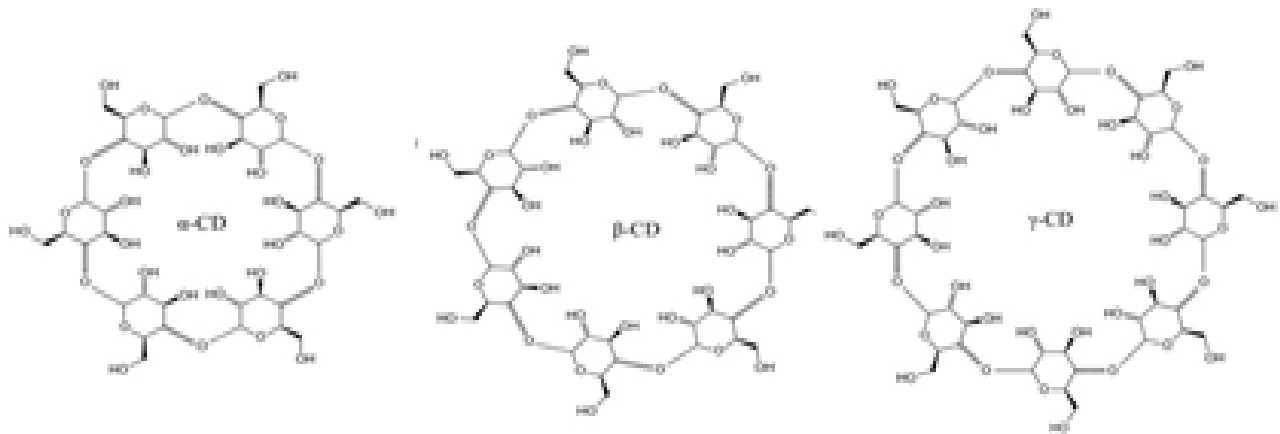

Fig. 1 Chemical structure of $\alpha, \beta$, and $\gamma$ cyclodextrin.

Cyclodextrin is also soluble in dimethyl sulfoxide, dimethylformamide, and pyridine. Cyclodextrins are of $(\alpha-1,4-)$ - linked $\alpha$-D-glucopyranose in the inner cavity, which is lipophilic in nature, and outer cavity hydrophilic-Glucopyranose as a chair formation shaped like cones (Fig. 1). At the same time, the cavity depends upon several glucose units in $\mathrm{CD}$. As units increase, the cavity will increase. $\beta-C D$ has 14 secondary hydroxyl groups with water and a narrow rim with seven primary hydroxyl groups from a bucket-like structure Wimmer (2003).

\section{Derivatives of cyclodextrin}

Cyclodextrin contains hydroxyl groups which help for grafting purposes, monomers are yielded by a crosslinking CD which forms linear or branched networks. Cyclodextrin derivatives used in pharmaceutical formulations are as-

Methylated CD- When the methyl group is substituted up to 14 groups for the $\beta$-CD ring, aqueous solubility also increases, which also directly depends on temperature. Low methylated substitution shows better biocompatibility when the temperature is elevated, while its partial substitution increases as the temperature are raised.

Hydroxypropyl CD- In pharmaceutical formulations $\beta$ and $\gamma$-CD $2 \mathrm{HP}$ derivatives are available as excipients. Good complex with neutral drug forms with $2 \mathrm{HP}-\beta-\mathrm{CD}$. HP- $\beta-\mathrm{CD}$ with DS of 2.7 or more show less water uptake, complex ability, and improved aqueous solubility.

Sulfobutyl ether (SBE) - Sulfonate and sulfoalkyl ether derivatives are formed when sultone in the presence of base is reacted with $\mathrm{CD}$. SBE- $\beta-\mathrm{CD}$ forms poor complex with anionic or neutral drugs due to anionic charge, while cationic drugs show a high binding constant and result in good complexation. SBE- $\beta-C D$ has an amorphous character which makes it highly water-soluble. Guest drug DS and alkyl ether chain length are responsible for SBE- $\beta C D$ derivatives binding capacity Jacob and Nair (2018).

\section{Amphiphilic nature of cyclodextrin}

CD's have inherent amphiphilic property (with an inner lipophilic core and an outer hydrophilicsurface)which aids to self-assembly resulting in formation of nanoparticles without any surfactant. For the formation of such supramolecular aggregates theCDs can be used as a carrier system for drug delivery. The amphiphilic CD can be obtained when the 6C aliphatic chain is grafted and linked with ester or amide group at the different faces of glucopyranose units. Limitation of parent $\mathrm{CD}$ can be solved by amphiphilic CD production, which 
also overcomes $\mathrm{CD}$ and hydrophobic drug interaction, increases $\mathrm{CD}$ and biological membrane interaction, and renders self-assembly capacity.

The amphiphilic CD is classified based on present charge on their surface, non-ionic, anionic, and cationic.

1) Non-ionic amphiphilic CD-In these types, CDs different length aliphatic chains are grafted on the primary or secondary face. Derivatives are named according to their structures-

a) Lollipop CD-here, a single aliphatic chain is grafted to 6- amino- $\beta C D$.

b) Cup and ball CD- here end of aliphatic chain linked with the voluminous group as test butyl group-prevents self-inclusion and provides higher solubility.

c) Medusa-like CD- C10 to C16 length aliphatic chain is grafted to the primary hydroxyl of CD molecule.

d) Skirt-shaped CD- contains $\alpha-C D, \beta-C D$, and $\gamma$-CD modified with aliphatic esters (C2 to C14) on secondary alcohol groups.

e) Bouquet-shaped CD- here 14polymethylene chains to 3monomethylated $\beta-\mathrm{CD}$ is grafted on each side of the $\mathrm{CD}$ ring, i.e., seven chains on both sides of it.

f) Cholesteryl CD- More complicated derivatives designed assuming that $\mathrm{CD}$ is a hydrophilic head and hydrophobic cholesterol position.

2) Cationic amphiphilic CD- These are synthesized and characterized by carrying an amino group as an ionic group in which hydroxyl groups of oligo (ethylene oxide). The cationic amphiphilic $\mathrm{CD}$ is also synthesized by modifying amido groups on CD's primary face with alkyl ethers were prepared. The cationic amphiphilic CD's structure property was derived from balancing thio alkyl chains and hydrophilic ethylene glycol oligomers. Ethylene glycol chains increase the colloidal stability of nano aggregates of the cationic amphiphilic $\mathrm{CD}$.
3) Anionic amphiphilic CD- These CDs are synthesized by linking sulfate or carboxyl groups that support and enhance anionic property on the secondary face's primary. Carboxyl CD amphiphiles prepared by attaching alkyl at 6 position and carboxyl methyl groups at 2 and 3 for sulphate amphiphilic CD 6 hydroxyl of an esterified $\mathrm{CD}$ at 2 and 3 position are sulfated, which makes them able to form a supramolecular aggregate in aqueous media Bilensoy and Hincal (2009), Varan et al. (2017).

\section{Self-assembly of cyclodextrin in aqueous solution}

Cyclodextrin molecules form self-assembly when present in an aqueous solution, this selfassembling tendency increases on inclusion complex formation and their concentration of inclusion complexes. Cyclodextrin aggregate easily if $\mathrm{CD}$ also forms an inclusion complex with guest moiety.

Cyclodextrin inclusion complex is formed when in cyclodextrin's hydrophobic cavity and lipophilic molecule get attached. CD aggregation also forms when $\mathrm{CD}$ guests can aggregate on themselves Messner et al. (2010). Inclusion complex forms by forces such as Vander Waal's forces, electrostatic interaction formation of Hydrogen bonds, and conformational strain release.

$\mathrm{CD}$ forms non-inclusion complexes where hydrogen bond formed due to hydroxyl group, which is present on the outer surface.

These aggregate shapes like spherical, rod-like, discs, reversible micelles, and these shapes will depend on solvent properties and solute molecule structure.

Many pharmaceuticals' preparations as nanoparticles hydrogels, liposomes, etc., are prepared by self-assembly property to overcome challenges like drug permeation and drug solubility, and permeable ability through biological members Ryzhakov et al. (2016).

\section{Toxicity}

Naturally occurring CD and CD's derivatives are accepted to be used as an excipient. Administrated CDs may cause toxicity, while it is seen that it is non-toxic when taken orally. Parenterally 
administered are eliminated without getting metabolize. Intravenous administration shows toxicity due to recrystallization potential to renal damage and nephrotoxicity. Sulphobutyl ether and hydroxypropyl CDs are found safe for parenteral formulations. Methylated CDs are found corrosive to tissue while RMBCD in low doses is safe to use in buccal delivery, but high dose produces an inflammatory effect Loftsson et al. (2005), Muankaew and Loftsson (2018).

\section{Advantages of cyclodextrin nanoparticles}

1) As a complexing agent for lipophilic drugs to increase their aqueous solubility.

2) Cyclodextrins control release modifier

3) Increase bioavailability

4) Increased stability as the drug lies in the cavity prevents oxidation for the photodiode.

5) Cyclodextrin masks the drug to prevent gastrointestinal irritation, reduce odor, andalso mask the taste.

6) Liquid dosages or oil can be converted to microcrystalline to improve handling.

7) Safe to use as only a low dose can be used to gain a therapeutic effect Jambhekar and Breen (2016), Woldum et al. (2008).

\section{Limitations/ disadvantages of cyclodextrin nanoparticles}

1) Diarrhea when taken orally.

2) May lead to nephrotoxicity when taken through the parenteral route.

3) Renal failure when taken through the intravenous route.

4) When taken through the buccal route, it may cause inflammation on a high dose.

5) Recrystallization in the bloodstream if taken intravenous route Lakkakula and Maçedo

Krause (2014), Loftsson (2014), Muankaew et al. (2020).

\section{Methods of preparation of cyclodextrin composites}

Cyclodextrin has non-polar cavity interaction of guests and wall of CD by hydrophobic interactions, Vander walls, and dipole-dipole interactions. There are many methods to prepare such complexes, which also depend upon guest molecule quality.
1) Physical mixture method-Cyclodextrin and drug are taken in 1:1 ratio, vigorously mixed in mortar for 3 hours, then passed through sieve and store in an airtight container Arias et al. (2000).

2) Kneading method- 1:1 ratio of cyclodextrin and drug to mortar to prepare a homogenous paste by using a solution of water and methanol in $1: 1$. Add drug to it in portions, continuous kneading for 3 hours. For proper consistency, add the solution in an appropriate quantity. In $45^{\circ}-50^{\circ}$ for 24 hours, paste is dried in a hot air oven. Sieved and stored Soniwala et al. (2005). This technique is done for a poor water-soluble guest molecule as a guest molecule is dissolved slowly during the procedure yields good complexes but not suitable for large-scale productionMadhuri et al. (2016), Marques (2010), Patel et al. (2007).

3) Solvent evaporation- Drug and $\beta-C D$ complexes were prepared by adding drug and $\mathrm{CD}$ in water using a round bottom flask; the solution is stabilized by keeping separately for three days. The solvent is dried out by using a rotator vacuum evaporator and lyophilization. Dry complex under vacuum and filter. Store solid mass in desiccators. Adhikari et al. (2019).

4) Co-precipitation method- In 1:1, drug and cyclodextrin are taken and dissolved in methanol and water, then the solution of the drug is added to CD solution dropwise. This mixture was stirred for 6 hours and then dried at $45^{\circ}-50^{\circ} \mathrm{C}$ for $48 \mathrm{hrs}$ collected and stored. This technique is used for non-water-soluble guest molecules but has poor yield due to competitive inhibition from an organic solvent Semalty et al. (2014a).

5) Spray drying- In deionized water $\mathrm{CD}$ and guest molecules are dissolved and dried by a spray dryer. Here temperature and sample feed speed can be regulated. As the temperature of $50^{\circ}-70^{\circ} \mathrm{C}$ is required, thermostable guest molecules are only used Semalty et al. (2014a).

6) Freeze-drying or lyophilization- Suitable technique for thermolabile or water-soluble 
guests. Cyclodextrin and drug dissolved in water while continuous stirring. Freeze-dried the obtained solution and washed the powder with organic solvent as benzene or chloroform rather than dried in a vacuum. This technique provides a good yield of the complex, so it can be used to produce complex on a large scale. Volatile oils can also be encapsulated in hydroxypropyl- $\beta$-CD Semalty et al. (2014a).

7) Microwave irradiation method- A solvent mixture of methanol and water is prepared of 1:1 v/v. The required amount of drug and equal amount $\mathrm{CD}$ were taken and triturated in a mortar and use the prepared solvent in minimum quantity. This mass is kept in a microwave oven to achieve dried mass and crushed to pass through a sieve for the desired sizeLoftsson et al. (2005), Loftsson et al. (2007)\& Semalty et al. (2014a).
8) Neutralization precipitation method- The drug is dissolved in alkaline solutions as sodium/ammonium hydroxide and mixed with $\mathrm{CD}$ are neutralized by agitating using hydrochloric acid solution until reaching equivalence point. The precipitate is formed, which is filtered, dried, and stored Patil et al. (2010).

\section{Evaluation study}

Cyclodextrin complex is evaluated for different parameters like yield, drug content, solubility, spectroscopy (UV, IR, NMR), microsopy (SEM), diffraction study and thermal analysis etc. (Table 2), Loftsson et al. (2005), Loftsson et al. (2007), Semalty et al. (2014a), Jacob and Nair (2018), Ghosh et al. (2011).

Table 2: Evaluation of cyclodextrin inclusion complexes
SN Study Instrument/Method

1. Physical Evaluation of color, appearance, and size. appearance

2. Percent practical Percent yield or method suitability is determined by using equation PY\% yield $=$ Practical mass $/$ Theoretical mass $($ drug + carrier $) \times 100$

3. Assay or Drug content estimation Transfer complex equivalent to $5 \mathrm{mg}$ of the drug to $25 \mathrm{ml}$ volumetric flask add methanol $10 \mathrm{ml}$ to it and keep for $5 \mathrm{~min}$ sonicating equipment. Made up the volume to $25 \mathrm{ml}$ and filter using Whatman filter paper in $25 \mathrm{ml}$ volumetric flask again makes up to $25 \mathrm{ml}$. Take $1 \mathrm{ml}$ of solution and dilute up to $10 \mathrm{ml}$ by distilled water and determined by spectrophotometry.

4. Solubility studies In a glass vial, take distilled water $5 \mathrm{ml}$ and add an excess of the drug to it, and each inclusion complex is added separately. Moreover, vials are shaken for $24 \mathrm{hrs}$ then filtered, appropriate dilutions are made using distilled water, and absorbance is recorded.

5. Thin-layer An appropriate amount of solvent is taken according to drug nature. Then chromatography inclusion complex with a reference sample of the pure drug is performed, and $\mathrm{RF}$ values were calculated.

6. UV visible UV spectra of inclusion complex and pure drug were recorded in spectroscopy

7. IR spectroscopy methanol a blank using methanol also will be run as reference. Fourier transform infrared spectroscopy is done to check the drug and carrier interaction by making its $\mathrm{kBr}$ pellets. The spectra were scanned in a proper frequency range.

8. X-ray diffraction X-ray diffraction evaluation is done of complex and of the drug for 
studies

Differential
scanning
calorimetric
(DSC)
(SEM) studies comparison. Diffraction patterns are recorded in the model as Bruner AXS, DH advanced model. The scanning rate is different for different drugs and complexes.

9. Scanning Electron To examine the morphology of formulation operating at the particular Microscopy accelerating voltage. Solid dispersions were carbon-coated to make

10. Dissolution electrically conductive, which produces an image in SEM. Inclusion complex sample equivalent to $5 \mathrm{mg}$ of the drug is taken for dissolution test apparatus and pure drug as control. The dissolution test is performed, and absorbance is measured by UV visible double beam spectrophotometer.

Samples are placed in a closed platinum crucible, and thermographs are generated and recorded.

\section{Applications of cyclodextrin and its complexes/nanoparticles}

The cyclodextrin can be used as a host for a wide range of molecules. These drug-CD inclusion complexes and nanoparticles are used in numerous fields as: -

\section{Food industry}

$\beta C D$ polymer to remove out the limonin and naringin, which cause bitterness in aqueous solution from orange and grapefruit, the polymer is extracted with an organic solvent. Cholesterol is removed from dairy products by crosslinking of $\beta C D$ with cholesterol which does not show any significant difference in texture, flavor, appearance, and taste even when stored for four weeks Jeon et al. (2012), Jung et al. (2008). To prevent flavor and taste oxidation and effect due to the environment can be encapsulated by forming inclusion complexes with CDs. So, by using CD encapsulation, degradation of flavor can be prevented, which is essential for consumer satisfaction and the life of food products Zhengyu (2013).

\section{Cosmetics}

$\mathrm{CD}$ is used to stabilize volatile and unstable compounds, reducing their vapor pressure, and increase solubility. CD prevents hydroquinone oxidation and increases its stability. In contrast, the whitening effect was found enhanced by Kojic acid when complexed with CDBuschmann and Schollmeyer (2002). CD solubilize and stabilize the product, which also prevents side effects of the active compound. Being a non-irritant and stable excipient can be used in cosmetics to improve quality Duchêne et al. (2005).

\section{Pharmaceuticals}

CD complexes increase solubility, bioavailability, stability, drug targeting, decrease the volatility of drugs, masking the taste or odor of drugs. Improve the physicochemical properties of dangerous drugs. Loading capacity is seen enhanced of nanoparticle congaing CD. CD's tendency toselfaggregate results to form nanoformulations which can be used to transport drugs across biological membranes as blood brain barrier. CD shows biocompatibility and inclusion capacity, which stables the drug, improved solubility, and masks the taste and odor. Due to such properties, there is a bright future when CD is used as an excipient in pharmaceuticals and nanomaterials Lakkakula and Maçedo Krause (2014).

\section{Analytical chemistry}

CD and its derivatives can be used as chiral stationary phases to separate enantiomers. CD's stationary phase's versatile nature can be used in three different chromatography modes, which are standard, reverse, and polar organic. So, the CD can play a role as a multi-model chiral stationary phase. Cyclodextrin complex is used for electrophoresis charged CD is used in capillary electrophoresis separation, which can also be used to monitor the drug and the impurities in the drug de Boer et al. (2000). 


\section{Amphiphilic Cyclodextrin Nanoparticles}

Cyclodextrin can spontaneously form nanoparticles due to the amphiphilic nature of cyclodextrin. The method of preparation of nanoparticles declares the formation of either nanospheres or nanocapsules. The standard techniques for the preparation of amphiphilic CD are as

\section{Solvent evaporation technique}

In the aqueous phase, the organic phase is emulsified by vigorous agitation. In this technique, initially, the water-immiscible organic phase containing the hydrophobic drug cyclodextrin, and oil is poured in waterwith agitation, and organic solvent application is evaporated by heat application. This technique requires reagents, chemicals that may cause toxicity. Toxicity may also bedue to the use of solvents as methylene chlorideBilensoy and Hincal (2009), Duchêne et al. (2005).

\section{Nanoprecipitation technique}

Amphiphilic CD nanospheres and nanocapsules prepared by the nanoprecipitation displacement method, which is based on interfacial turbulence between the miscible organic phase and an aqueous phase due to the precipitation of the organic phase, ethanol or acetone can be used, and for non-solvent ultrapure water is used such two different phases are necessary for this method. By this method, the surfactant is not required smooth surface nanoparticles are obtainedBilensoy and Hincal (2009), Varan et al. (2017).

\section{Drug loading}

The drug can be loaded into amphiphilic cyclodextrin complexes / nanoparticles by different techniques to bind or encapsulate drug into cyclodextrin nanoparticles by-

1. Conventional loading method-The solution containing drugs is directly added to the organic phase in the nanoprecipitation technique process.

2. Pre-loading method- Drug and cyclodextrin complexes are directly used to prepare cyclodextrin nanoparticle complexes. Here no extra drug is loaded.

3. High loading method- In this method, excess of drug solution, that is dissolved in the organic phase, is added further to drug: amphiphilic cyclodextrin complexes.

The free drug can be separated by applying centrifugation, and entrapped drug quantity is a mathematically expressed ratio of determining drug quantity over initial cyclodextrin quantityMemişoğlu et al. (2003).

\section{Cyclodextrin nanoparticles in drug delivery}

Therapeutic drug delivery is directly proportional to the quantity of drug concentration on the site. Body barriers not only act as the protective layer for microorganisms, but drug absorption is also restricted due to barriers. Cyclodextrin can act as an excipient for enhancing permeability for drug delivery, especially in topical and oral drug delivery Muankaew et al. (2020). Cyclodextrin nanoparticles formulation can be used for drug delivery main routes are as follows-

\section{Ocular drug delivery}

Nanoparticles' role in ocular targeting is seen in many contrasts, so the formulations are prepared in the form of nanoparticles that can show specific targeting on every site of the eye Diebold and Calonge (2010). Formulations that contain $\alpha$ and $\gamma$ $\mathrm{CD}$ enhances the solubility of the drug, which is due to the presence of $\alpha \mathrm{CD}$, and $\gamma \mathrm{CD}$ helps in increasing self-aggregation tendency of the formulation. No side effects such as irritation or inflammation are seen, formulation with $\alpha$ and $\gamma$ $\mathrm{CD}$ as excipients are safe to be used Jóhannsdóttir et al. (2017).

The outer hydrophilic surface responsible for solubility in the eye and hydrophobic cavity form inclusion complex and results in improving the entrapped lipophilic molecule's bioavailability, and the formulation was free of any irritation Pontillo and Detsi (2019).

With increasing solubility and aggregation, property sustained release of drugs can be achieved corneal permeation is enhanced by complexing HP- $\beta C D$ and drug in the form of drops or gel. The nanosized formulation can deliver drugs effectively to intraocular tissues, and drug release also sustained Elmotasem and Awad (2020). 
Solubility is seen enhanced by binding drug with $\mathrm{CD}$, and a higher drug release rate is found while on preparing nanoparticle of it by using chitosan as polymer a sustained release of drug is seen, which is convenient to use by the patient and the formulation is found free of inflammation, irritation, or any side effects. So, nanocarriers of $\mathrm{CD}$ can be used for ocular drug delivery as it solubilizes fast, the drug's bioavailability is improved, the drug release time is prolonged, easy for application or use drug dosing frequency is reduced Zhang and Liu (2016).

\section{Parenteral drug delivery}

Cyclodextrin is used to prepare formulations for injectable purposes to raise the solubility of poorly soluble drugs. Today there are many injectable solutions present in the market for both intravenous and intramuscular administration. These preparations can also be modified to carry proteins and peptides to it Jambhekar and Breen (2016). CD drug complex shows low or medium stability value, and drug released by simple dilution when taken by the intravenous route. Protein binding of drug decreases, so ultimately, CD-NPS drug complex is not enough to have a good volume of distribution of drugs. Renal clearance is also seen enhanced Loftsson (2014).B-CD show binding with cholesterol that may result in nephrotoxicity. $\alpha$ and methylated $\beta$ $\mathrm{CD}, \boldsymbol{\gamma}$-CD causes renal toxicity when administered parenterally. Chemically modified CDs such as HP- $\beta$-CDs and SBE- $\beta$-CDs are used as an excipient in parenteral products. It is also found safe to be used in intravenous preparations, where elimination from systematic circulation is fast by renal excretion Jambhekar and Breen (2016).

Hydroxyalkylated CD improves solubility and the bioavailability of drug and free of toxicity; drug level in plasma found higher when the drug is complexed with HP- $\beta-C D$, higher plasma level due to enhanced solubility in water, while it is found that pharmacokinetic parameters are equal to oral administration as compared to intramuscular administration. Drug irritation is also reduced by complex, which shows the 2 HP-
$\beta$-CD can be used in pharmaceuticals to formulate parenteral products Yoshida et al. (1990). HP- $\beta$ $\mathrm{CD}$ and SBE $\beta-\mathrm{CD}$ can be used for forming a complex with drugs for parenteral formulations; they are safe to use. Additionally, these formulations can be sterilized by applying gamma irradiation, which sterilizes the formulation and reduces the complex size; gamma irradiation does not alter complex characteristics MemisogluBilensoy and Hincal (2006).

\section{Oral drug delivery}

Cyclodextrin complex is used to improve drug solubility, bioavailability to reduce irritation, taste, and odor of drug, GIT also stabilizes drug at the absorption site. Cyclodextrin complexes are seen to have enhanced absorption with uniformity of poorly water-soluble drugs, that are easy to administer through the buccal or sublingual route. The drug is also prevented by GIT content by complexation with $\mathrm{CD}$. $\mathrm{CD}$ or its derivatives are safe to be taken orally as there is no CD absorption through GIT Challa et al. (2005).

Drugs for oral delivery can be prepared by the HP $\beta$-CD drug complex, which improves stability and absorption of drugs like insulin which can further encapsulate mucoadhesive polymethacrylic acidbased nanoparticles high residence time and reduces proteolytic degradation Sajeesh and Sharma (2006). Drugs' first-pass metabolism can be avoided by $\mathrm{CD}$ complex. When the drug is complexed with carboxymethyl $\beta-C D$ grafted chitosan nanoparticles, it shows sustained release. Protein drug delivery is possible by CMCD- $\boldsymbol{\gamma}-\mathrm{CD}$ nanoparticles preparationChaudhury and Das (2011), Song et al. (2017).

Studies confirmed that cyclodextrin nanoparticles could be used for peptide and protein orally administer. The nanoparticle can target the specific site and cyclodextrin provide the ability to carry hydrophobic drug with it, so finally, both combination results in a formulation capable of successful oral delivery with low toxicity Kanwar et al. (2011).

\section{Transdermal drug delivery}

Cyclodextrin can be used to enhance drug release and as a permeation enhancer that stabilizes the drug in the formulation and prevents local 
irritation at the place of the absorptive site. Hydrophilic cyclodextrin can improve the lipophilic drug release rate from the hydrophilic aqueous vehicle. Cyclodextrin provides free drugs at the barrier surface, which dissolves out by cyclodextrin complexes at the absorption site. Cyclodextrin reduces drug degradation in topical preparation and skin irritation due to the drug is alleviatedChalla et al. (2005). Excipients used in dermal drug formulation as permeation enhancers are alcohol or fatty acids that temporarily prohibit the stratum corneum's barrier property. At the same time, hydrophilic cyclodextrin can penetrate without any effect on barrier property, and the permeable amount is meager. Cyclodextrin is seen to reduce the amount of hydrocortisone permeability in w/o cream while enhanced release from $\mathrm{o} / \mathrm{w}$ cream. So, cyclodextrin can be used in the UV absorbing compound by adding 2hydroxypropyl $\beta \mathrm{CD}$ in excess prevent sunscreen permeation to the skin, and enhances the protective effect from UV for a long durationLoftsson et al. (2005). The nanoparticle of the cyclodextrin complex was found to have a smooth and proper distribution of the drug on the applied area and shows high drug entrapment efficiency. Nanoparticles initially burst the drug and latterly releases in a controlled phase, which is due to complex with $\beta \mathrm{CD}$. The permeation of drug is enhanced due to nanosize of the formulation so a successful formation can be prepared by complexing drug with cyclodextrin loaded in chitosan nanoparticles Khalil et al. (2012). CD complex with mocoxazole was found to increase solubility and stability of micoxazole.

\section{Requirement of cyclodextrin in the pharmaceutical industry}

While it takes more than 100 years to enable cyclodextrin as a pharmaceutical excipient, cyclodextrin's ability to amplify aqueous bioavailability, solubility, and stability of drug proved for formulations of cyclodextrin complexes with the drug.

Cyclodextrin forms non-inclusion complexes and shows aggregation among itself and with guest molecules. This property leads to form dispersed drug delivery system as nanoparticles or microparticles. Thus, cyclodextrin-based technologies are constantly developing in the pharma industry.

\section{Future opportunities and challenges}

CD was discovered in the 19th century, but nowadays it is widely used excipient due to many benefits. CD-based technologies are developing to extend the stability, solubility, targeting, and bioavailability of drugs. CD acts as a complexing agent for hydrophobic drugs that increase their solubility, so it can be used for class II and class IV drugs (of BiopharmaceuticalClassification System). CD and its complexes can also restrict the drug anddeliver the drug in a controlled manner that can be further modified to sustained or controlled release formulations. Further, CD can be used for masking the odor, taste and to prevent first-pass metabolism CD can also be used for volatile oils to provide protection and stabilizationHadian et al. (2018), Semalty et al. (2014a), Woldum et al. (2008).

Modification of $\mathrm{CD}$ by grafting different chains into it may lead to improvement in its amphiphilic nature. Thisaids the tendency in CDs to self-aggregate among themselves without use of any surfactant or any chemical, and hence can be used to formulate nano preparation for drug targeting purposes. In pharmaceutical sciences, chemical flexibility of CD's structure, design of versatile drug carrier as for both hydrophobic and hydrophilic drugs provides a wide range of derivative CDs that can be used as an excipient for many purposes in the pharmaceutical fieldLoftsson and Duchêne (2007).

With respect to drug targeting, $\mathrm{CD}$ based nanoformulations can be explored. The CD's tendency to form self-aggregate may lead to nanostructures that make it easy to deliver protein, antibiotics, and vaccines a specific target. Cyclodextrin increases the solubility of lipophilic drugs, which contribute more to deliver drugs precisely. There are aroundthirty different pharmaceutical formulations in the market, and more formulations are to be formulated to increase the ability and efficiency of the drug,Messner et al. (2010). 


\section{Conclusion}

To deal with the chemical and physical instability of the drugscyclodextrins has been proven to be of potential value in dosage form and drug delivery system development. CD also prevents local irritation and alleviates undesirable drug properties. CD's self-aggregation property of forming nanoformulationsis being used in developing novel drug deliverysystems and tumor targeting. Solubility (as well as the permeability) of drugs (of Class II and IV drugs of biopharmaceutical classification system) is enhanced by $\mathrm{CD}$ complexes/ nanoparticles of drugs. The $\mathrm{CD}$ based formulations can be used to achieve the desired therapeutic effect on the body.CD nanoformulation can be considered as a new developing phase where many aspectsare remaining to be explored.

\section{Acknowledgement}

Authors are thankful to UGC-DAE Consortium for Scientific Research, Indore, India for providing the research grant and fellowship under CRS (CRS-310).

\section{References}

Adhikari L, Semalty M, Naruka PS, Aswal VK, Semalty A (2019) Binary complexes of glimepiride with $\beta$-cyclodextrin for improved solubility and drug delivery. Indian Drugs 56(03): 55.

Arias MJ, Moyano JR, Muñoz P, Ginés JM, Justo A, Giordano F (2000) Study of omeprazole$\gamma$-cyclodextrin complexation in the solid state. Drug Dev. Ind. Pharm. 26(3): 253-259.

Bilensoy E, Hincal AA (2009) Recent advances and future directions in amphiphilic cyclodextrin nanoparticles. Expert. Opin. Drug. Deliv. 6(11): 1161-1173.

de Boer T, de Zeeuw RA, de Jong GJ, Ensing K (2000) Recent innovations in the use of charged cyclodextrins in capillary electrophoresis for chiral separations in pharmaceutical analysis. Electrophoresis 21(15): 3220-3239.
Buschmann HJ, Schollmeyer E (2002) Applications of cyclodextrins in cosmetic products: A review. J. Cosmet. Sci. 53(3): 185-191.

Challa R, Ahuja A, Ali J, Khar RK (2005) Cyclodextrins in drug delivery: An updated review. AAPS PharmSciTech 6(2): E329E357.

Chaudhury A, Das S (2011) Recent advancement of chitosan-based nanoparticles for oral controlled delivery of insulin and other therapeutic agents. AAPS Pharm SciTech 12(1): 10-20.

Coltescu AR, Butnariu M, Sarac I (2020) The Importance of Solubility for New Drug Molecules. Biomed. Pharmacol. J. 13(2): 577-583.

Diebold Y, Calonge M (2010) Applications of nanoparticles in ophthalmology. Prog. Retin. Eye Res. 29(6): 596-609.

Duchêne D, Ponchel G, Bochot A (2005) New uses of cyclodextrins. Eur. J. Pharm. Sci. 25: S1-S226.

Elmotasem H, Awad GEA (2020) A stepwise optimization strategy to formulate in situ gelling formulations comprising fluconazolehydroxypropyl-beta-cyclodextrin complex loaded niosomal vesicles and Eudragit nanoparticles for enhanced antifungal activity and prolonged ocular delivery. Asian J. Pharm. Sci. 15(5): 617-636.

Ghosh A, Biswas S, Ghosh T (2011) Preparation and Evaluation of Silymarin $\beta$-cyclodextrin Molecular Inclusion Complexes. J. Young. Pharm. 3(3): 205-210.

Hadian Z, Maleki M, Abdi K, Atyabi F, Mohammadi A, Khaksar R (2018) Preparation and Characterization of Nanoparticle $\quad \beta$-Cyclodextrin:Geraniol Inclusion Complexes. Iran J. Pharm. Res. 17(1): 39-51.

Hedges A (2009) Chapter 22 - Cyclodextrins: Properties and Applications. BeMiller J, Whistler R, eds. Starch (Third Edition). Food Science and Technology. (Academic Press, San Diego), pp.833-851. 
Jacob S, Nair AB (2018) Cyclodextrin complexes: Perspective from drug delivery and formulation. Drug Dev. Res. 79(5): 201-217.

Jambhekar SS, Breen P (2016) Cyclodextrins in pharmaceutical formulations II: solubilization, binding constant, and complexation efficiency. Drug Discov. Today 21(2): 363-368.

Jeon SS, Lee SJ, Ganesan P, Kwak HS (2012) Comparative study of flavor, texture, and sensory in cream cheese and cholesterolremoved cream cheese. Food Sci. Biotechnol. 21(1): 159-165.

Jóhannsdóttir S, Kristinsson JK, Fülöp $Z$, Ásgrímsdóttir G, Stefánsson E, Loftsson T (2017) Formulations and toxicologic in vivo studies of aqueous cyclosporin A eye drops with cyclodextrin nanoparticles. Int. J. Pharm. 529(1-2): 486-490.

Jung TH, Ha HJ, Ahn J, Kwak HS (2008) Development of cholesterol-reduced mayonnaise with crosslinked $\beta$-cyclodextrin and added phytosterol. Korean J. Food Sci. Anim. Resour. 28(2): 211-217.

Kanwar JR, Long BM, Kanwar RK (2011) The use of cyclodextrins nanoparticles for oral delivery. Curr. Med. Chem. 18(14): 20792085.

Khalil SKH, El-Feky GS, El-Banna ST, Khalil WA (2012) Preparation and evaluation of warfarin- $\beta$-cyclodextrin loaded chitosan nanoparticles for transdermal delivery. Carbohydr. Polym. 90(3): 1244-1253.

Kreaz ARM, Abu-Eida EY, Eros I, Kata M (1999) Freeze-dried complexes of furosemide with $\beta$-cyclodextrin derivatives. J. Incl. Phenom. Macrocycl. Chem. 34(1): 39-48.

Lakkakula JR, Maçedo Krause RW (2014) A vision for cyclodextrin nanoparticles in drug delivery systems and pharmaceutical applications. Nanomedicine 9(6): 877-894.

Loftsson T (2014) Self-assembled cyclodextrin nanoparticles and drug delivery. J. Incl. Phenom. Macrocycl. Chem. 80(1): 1-7.

Loftsson T, Duchêne D (2007) Cyclodextrins and their pharmaceutical applications. Int. J. Pharm. 329(1-2): 1-11.
Loftsson T, Jarho P, Másson M, Järvinen T (2005) Cyclodextrins in drug delivery. Expert Opin. Drug. Deliv. 2(2): 335-351.

Loftsson T, Saokham P, Sá Couto AR (2019) Selfassociation of cyclodextrins and cyclodextrin complexes in aqueous solutions. Int J Pharm 560: 228-234.

Mehar M, Mukesh P, Lokesh A, Ajay S, Mona S (2016) Effect of Hydrophilic excipients on cyclodextrin complexes of acyclovir in improving solubility, dissolution and permeability. Lett. Drug Des. Discov. 13(8): 771-780.

Marques HMC (2010) A review on cyclodextrin encapsulation of essential oils and volatiles. Flavour. Fragr. J. 25(5): 313-326.

Memişoğlu E, Bochot A, Şen M, Duchêne D, Hincal AA (2003) Non-surfactant nanospheres of progesterone inclusion complexes with amphiphilic $\beta$-cyclodextrins. Int. J. Pharm. 251(1): 143-153.

Memisoglu-Bilensoy E, Hincal AA (2006) Sterile, injectable cyclodextrin nanoparticles: Effects of gamma irradiation and autoclaving. Int. J. Pharm. 311(1): 203-208.

Messner M, Kurkov SV, Jansook P, Loftsson T (2010) Self-assembled cyclodextrin aggregates and nanoparticles. Int. J. Pharm. 387(1): 199-208.

Muankaew C, Loftsson T (2018) CyclodextrinBased Formulations: A Non-Invasive Platform for Targeted Drug Delivery. Basic Clin. Pharmacol. Toxicol. 122(1): 46-55.

Muankaew C, Saokham P, Jansook P, Loftsson T (2020) Self-assembly of cyclodextrin complexes: detection, obstacles and benefits. Pharmazie 75(7): 307-312.

Mura P (2014) Analytical techniques for characterization of cyclodextrin complexes in aqueous solution: A review. J. Pharm. Biomed. Anal. 101: 238-250.

Patel HM, Suhagia BN, Shah SA, Rathod IS, Parmar VK (2007) Preparation and characterization of etoricoxib-betacyclodextrin complexes prepared by the kneading method. Acta Pharm. 57(3): 351359. 
Patil J, Kadam D, Marapur S, Kamalapur M (2010) Inclusion complex system; a novel technique to improve the solubility and bioavailability of poorly soluble drugs: a review. Int. J. Pharm. Sci. Rev. Res. 2(2): 2934.

Peñalva R, Esparza I, Morales-Gracia J, González-Navarro CJ, Larrañeta E, Irache JM (2019) Casein nanoparticles in combination with 2-hydroxypropyl- $\beta$ cyclodextrin improves the oral bioavailability of quercetin. Int. J. Pharm. 570: 118652.

Pontillo ARN, Detsi A (2019) Nanoparticles for ocular drug delivery: modified and nonmodified chitosan as a promising biocompatible carrier. Nanomedicine 14(14): 1889-1909.

Ryzhakov A, Do Thi T, Stappaerts J, Bertoletti L, Kimpe K, Sá Couto AR, Saokham P, et al. (2016) Self-Assembly of Cyclodextrins and Their Complexes in Aqueous Solutions. $J$. Pharm. Sci. 105(9): 2556-2569.

Sajeesh S, Sharma CP (2006) Cyclodextrin-insulin complex encapsulated polymethacrylic acid based nanoparticles for oral insulin delivery. Int. J. Pharm. 325(1-2): 147-154.

Semalty A (2014a) Cyclodextrin and phospholipid complexation in solubility and dissolution enhancement: A critical and meta-analysis, Expert Opin. Drug. Deliv. 11(8):1255-1272.

Semalty A, Tanwar YS, Semalty M (2014b) Preparation and characterization of cyclodextrin inclusion complex of naringenin and critical comparison with phospholipid complexation for improving solubility and dissolution. J. Therm. Anal. Calorim. 115(3): 2471-2478.

Song M, Li L, Zhang Y, Chen K, Wang H, Gong $\mathrm{R}$ (2017) Carboxymethyl- $\beta$-cyclodextrin grafted chitosan nanoparticles as oral delivery carrier of protein drugs. React. Funct. Polym. 117: 10-15.

Soniwala MM, Patel PR, Mansuri NS, Parikh RK, Gohel MC (2005) Various approaches in dissolution enhancement of rofecoxib. Indian J. Pharm. Sci. 67(1): 61-64.
Szejtli J (1998) Introduction and general overview of cyclodextrin chemistry. Chem. Rev. 98(5): 1743-1754.

Varan G, Varan C, Erdoğar N, Hıncal AA, Bilensoy E (2017) Amphiphilic cyclodextrin nanoparticles. Int. J. Pharm. 531(2): 457469.

Wimmer T (2003) Cyclodextrins. Ullmann's Encyclopedia of Industrial Chemistry. (American Cancer Society).

Woldum HS, Larsen KL, Madsen F (2008) Cyclodextrin Controlled Release of Poorly Water-Soluble Drugs from Hydrogels. Drug Deliv. 15(1): 69-80.

Yoshida $\mathrm{A}$, Yamamoto $\mathrm{M}$, Itoh $\mathrm{T}$, Irie $\mathrm{T}$, Hirayama F, Uekama K (1990) Utility of 2hydroxypropyl-beta-cyclodextrin in an intramuscular injectable preparation of nimodipine. Chem. Pharm. Bull. (Tokyo) 38(1): 176-179.

Zhang J, Liu H (2016) A novel approach to preparing polystyrene/Fe3O4 multihollow microspheres with porous walls. Coll. Polym. Sci. 294(11): 1755-1763.

Zhengyu J (2013) Cyclodextrin Chemistry: Preparation And Application (World Scientific / Chemical Industry Press, China). $* * * * * * *$ 